\title{
A Neural-based Minutiae Pair Identification Method for Touch-less Fingerprint Images
}

\author{
Ruggero Donida Labati, Vincenzo Piuri, IEEE, Fellow, Fabio Scotti, IEEE, Member \\ Department of Information Technologies \\ Università degli Studi di Milano \\ Milano, 20122, Italy. \\ ruggero.donida@unimi.it,vincenzo.piuri@unimi.it, fabio.scotti@unimi.it
}

\begin{abstract}
Contact-based sensors are the traditional devices used to capture fingerprint images in commercial and homeland security applications. Contact-less systems achieve the fingerprint capture by vision systems avoiding that users touch any parts of the biometric device. Typically, the finger is placed in the working area of an optics system coupled with a CCD module. The captured light pattern on the finger is related to the real ridges and valleys of the user fingertip, but the obtained images present important differences from the traditional fingerprint images. These differences are related to multiple factors such as light, focus, blur, and the color of the skin. Unfortunately, the identity comparison methods designed for fingerprint images captured with touch-based sensors do not obtain sufficient accuracy when are directly applied to touch-less images. Recent works show that multiple views analysis and 3D reconstruction can enhance the final biometric accuracy of such systems. In this paper we propose a new method for the identification of the minutiae pairs between two views of the same finger, an important step in the 3D reconstruction of the fingerprint template. The method is divisible in the sequent tasks: first, an image preprocessing step is performed; second, a set of candidate minutiae pairs is selected in the two images, then a list of candidate pairs is created; last, a set of local features centered around the two minutiae is produced and processed by a classifier based on a trained neural network. The output of the system is the list of the minutiae pairs present in the input images. Experiments show that the method is feasible and accurate in different light conditions and setup configurations.
\end{abstract}

Index Terms-neural-networks, touch-less fingerprint, contactless fingerprint, minutiae matching

\section{INTRODUCTION}

Fingerprint images captured by touch-less sensors are very different from fingerprint images captured by traditional touchbased sensors. Typically, such kind of images are more noisy, the ridge pattern is less visible and the color of the sky can significantly change. Moreover, defocusing problems as well as blur effects due to the movements of the finger can be present. For this reason, in a biometric system designed for touch-less fingerprint images, the feature extraction and image processing methods must be carefully selected and tuned taking into account the presence of the specific noise type related to the biometric samples.

In the literature, there are several biometric recognition systems designed for touch-less fingerprint images. The majority of these systems are based on multiple finger images [1 $3]$, or single images $[4,5]$. Unfortunately, the real accuracy

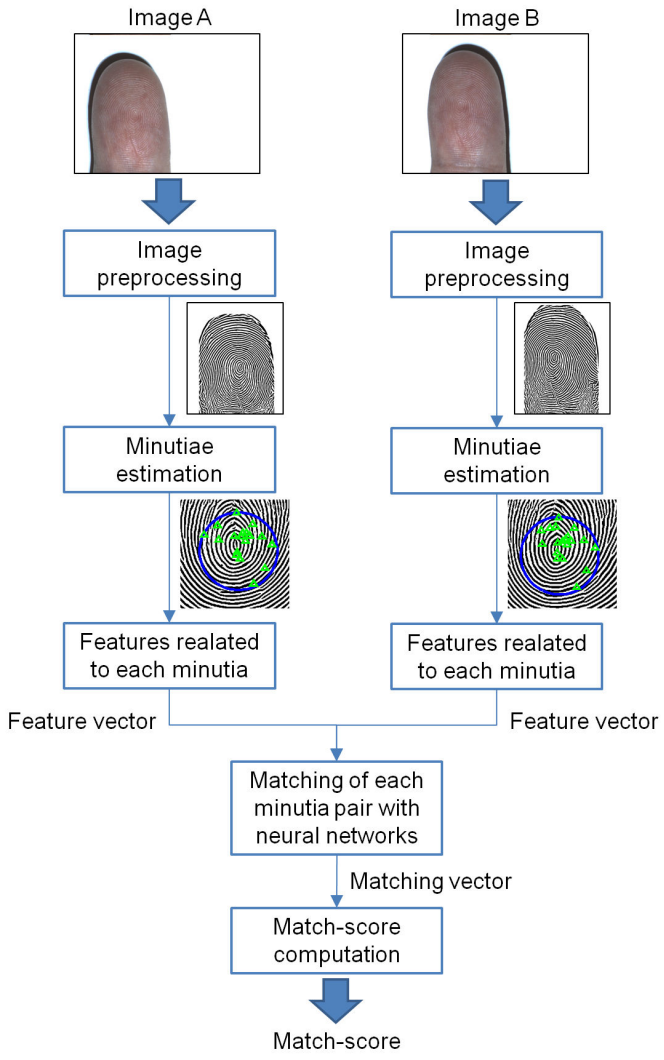

Fig. 1. Schema of the proposed method.

of such system is not comparable to the one achieved by touch based fingerprint recognition systems. In many cases, the performances are far to be sufficient for real applicative contexts, especially for systems working with a single image of the finger. Preliminary results in this research field show that systems based on multiple views of the finger (including multiple view and structured light three-dimensional systems) show a more accurate behavior, but, on the other side, they need more complex setups (more cameras and /or special illumination systems) and specific software modules to deal with the complexity of their image inputs.

In this paper we refer to three-dimensional systems that localize the minutiae in the three-dimensional space by two or more views of the finger (Fig. 1). The images related to 


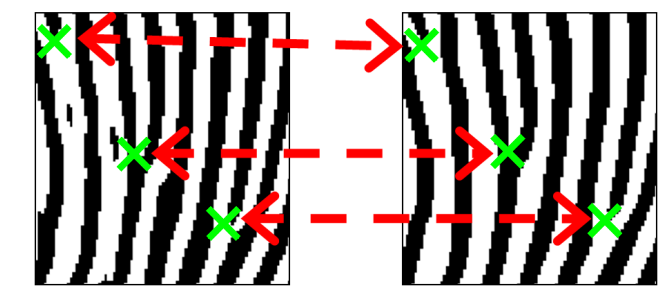

Fig. 2. Example of three minutiae pairs in two different views of the same finger.

the different views can be captured simultaneously by using $N$ cameras, or in a time sequence by using a single camera that captures a frame sequence of the finger by different point of views. In many of these applications, the surface points of the finger are reconstructed in the three-dimensional space by applying the triangulation technique, using candidate points that have been identified in each frame, and estimating the correspondence of these points in the different images. This step is the more crucial in these three-dimensional reconstruction methods, since errors in the identification of the same point in the different images lead to inaccurate reconstructions and hence to a wrong behavior of the subsequent biometric system.

In particular, in this paper we present a new minutiae matcher based on neural networks that is designed for touchless fingerprint images. This method is capable to identify two candidate sets of minutiae from two touch-less images, and then select a final list of minutiae pairs. Fig. 2 shows an example of the input and output of the proposed system, where the arrows show three identified pairs of minutiae, which are ready to be transformed in a single three-dimensional point.

Considering two minutia points extracted from two fingerprint images, the proposed system is capable to estimate if these two points belong to the same minutiae in the real finger. In our approach, the images captured by touch-less sensors are first enhanced and segmented, in order to improve the visibility of the ridge pattern and reducing the presence of noise. Second, the minutiae positions are estimated with an algorithm that is well known in the literature. Then, a set of features capable to describe the local discriminative intensity pattern is computed for each minutia. Last, the final comparison is processed by using a trained neural network that evaluates the differences between the local features of the candidate minutiae pairs.

The paper is structured as follows. In Section II, the state of the art is briefly commented. In Section III, the proposed method is presented and detailed. In Section IV, the creation of the training and test datasets is described, it is presented the design of the neural networks, and the overall results are given and compared with other techniques present in the literature. In the last section, the overall behavior of the proposed method and the future work are discussed.

\section{Previous WORK}

In the literature, most of the identity comparison methods based on the fingerprint biometric trait use information related to minutiae. These methods typically consider each minutia as a 3-tuple composed by the coordinates and the angle of the ridge in the minutia point. Usually, the minutiae type is not considered because different pressures of the finger on the acquisition sensor can modify bifurcation in termination and vice versa. A common approach encompasses the registration of two minutiae sets and compute the match-score by counting the number of matched minutiae pairs. Examples of methods based on the minutiae pattern matching are the software Bozorth3 of the National Institute of Standards and Technology (NIST) [5], and the method proposed in [6]. A different approach considers the structural matching of the minutiae. The methods related to this approach compute a graph that represents the structure of the fingerprint, then the match-score between two fingerprint is computed by applying inexact graph matching algorithms. For example, the method proposed in [7] is based on graphs obtained by the Delaunay triangulation, and the method in [8] by the Voronoi diagram. Other methods in the literature use supplementary information, for example, the CilinderCode [9], and information obtained by applying Gabor filters in the areas near to the minutiae [10]. There are also many fingerprint recognition methods based on neural networks. Some of these methods use information related to the minutiae coordinates [11, 12], but most of them are based on more complex features [13 - 15].

The identity comparison methods designed for images captured by classical touch-based sensors cannot be directly applied on touch-less fingerprint images. For this reason, the biometric recognition systems in the literature designed for touch-less fingerprint images apply image processing algorithms for improving the image quality and then compute the match-score between two images by applying classical algorithms. It is possible to distinguish methods that computes three-dimensional models [1, 2], fingerprint images obtained by the mosaic of a set of images [3], and methods based on single fingerprint images $[4,5]$. The methods based on multiple finger images estimate rolled-equivalent images, and then apply classical identity comparison methods. These methods obtain good performances in terms of accuracy but are computationally expansive. The methods based on single images apply image enhancement techniques, and then use classical identity comparison methods. Usually, the required computational time is less than the time required by the methods based on three-dimensional models, but the obtained accuracy is lower.

\section{The Proposed Method}

In this paper, we propose a method for the identification of minutiae pairs in fingerprint touch-less images. In particular, the novelty is twofold: an innovative composition of features is presented and the application of neural networks is introduced. In the following we describe the specific enhancement algorithm designed for contactless fingerprint image, the processing of the local feature, and the design of the neural networks. 


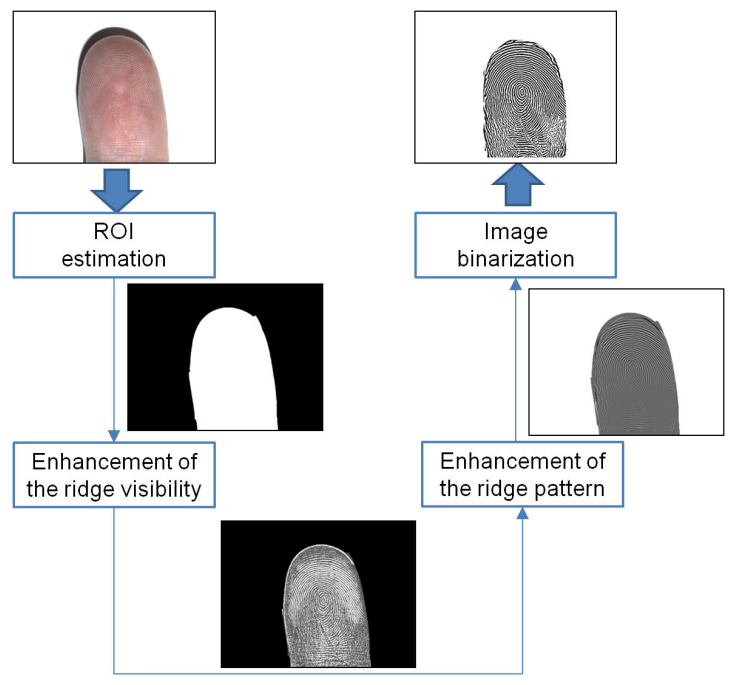

Fig. 3. Schema of the proposed image preprocessing and ROI selection method.

\section{A. Enhancement of the images ROI selection}

The objectives of this step are the enhancement of the fingertip contrast, the noise reduction, and the definition of the region of interest (ROI). The proposed method is similar to the method described in [16]. This method can be divided in four distinct steps:

- ROI estimation;

- enhancement of the ridge visibility;

- enhancement of the ridge pattern;

- image binarization.

Fig. 3 shows the schema of the proposed method.

The ROI estimation is performed by using an algorithm based on the local standard deviation of the image, which is described in [16], and by removing the shadow of the finger by a threshold operation.

The second step of the proposed method is the enhancement of the ridge visibility. First, the background image $I_{B}$ is estimated by applying a morphological open operation with a mask $s$ to the image $I$. Then, the background is removed, obtaining the image $I_{R}$. For reducing the noise effect present in the images, we perform a nonlinear equalization by processing the logarithm of $I_{R}$. An image $I_{L}$ is computed as $I_{L}(x, y)=\log \left(I_{R}(x, y)\right)$.

The third step is the enhancement of the ridge pattern. Similarly to the work proposed in [16, 17], this algorithm estimates a ridge frequency map, a ridge orientation map, and applies to the image a set of Gabor filters tuned according to the computed maps. The obtained result is the image $I_{E}$.

The last step of the proposed method consists in the image binarization. This step is necessary to reduce the noise present in the edges of the finger surface in the image $I_{E}$ and to correctly estimate the minutiae points. For obtaining a uniform contrast between ridges and valleys, we compute the logarithm of $I_{E}$ as $I_{I}(x, y)=\log \left(I_{E}(x, y)\right)$. Then, the histogram $H$ of $I_{I}$ is computed, and a binary image of the ridge pattern $I_{B}$ is obtained as follow

$$
I_{B}(x, y)=\left\{\begin{array}{ll}
0 & \text { if } I_{I}(x, y) \leq \underset{i}{\operatorname{argmax}}(H(i)) \\
1 & \text { otherwise }
\end{array} .\right.
$$

The image $I_{B}$ is used only for the minutiae point estimation. The other features are extracted from $I_{E}$.

\section{B. Minutiae selection and extraction of the local feature set}

The minutia positions are estimated by the software Mindtct of the NIST [5]. This software is one of the most used in the literature for the estimation of the minutia points. The proposed enhancement allow to use Mindtct also in the case of contact-less fingerprint images. The output of the method are the coordinates of the minutiae, the angle $\theta$ of the ridge related to each minutia, and the quality of the minutia points.

For a fingerprint image $A$, three different matrices of local feature $\left(M_{A}, F_{A}, H_{A}\right)$ are computed. The values of these matrices are related the local areas centered in each minutia point $i$.

The matrix $M_{A}$ is related to the data obtained by the software Mindtct and is composed by the sequent elements.

- $M_{A}(i, 1)$ : the $x$-coordinate of the point.

- $M_{A}(i, 2)$ : the $y$-coordinate of the point.

- $M_{A}(i, 3)$ : the minutia angle $\alpha$ normalized considering the median ridge orientation

$$
\alpha=\beta+\theta,
$$

where $\beta$ is the median value of the area of the fingerprint orientation image that appertains to the ROI.

- $M_{A}(i, 4)$ : the estimated quality of the minutia.

The matrix $F_{A}$ is composed by $N_{F}$ elements obtained by the computation of the template Fingercode around each minutia point. This template is obtained by applying the method described in [18]. This method is divisible in the sequent steps.

- A local ROI is defined as a circle with fixed size (height $H$ ) centered in the coordinates of the considered minutia point $\left(x_{i}, y_{i}\right)$.

- The local ROI is tasseled in $N_{R}$ rings and $N_{A}$ arcs, obtaining $N_{S}=N_{R} \times N_{A}$ sectors $S_{i}$.

- A set of $N_{F}$ Gabor filters with different directions is computed and applied to the local area of the fingerprint image. The obtained result consists in $N_{F}$ filtered images $T_{k \theta}$. In the spatial domain, a symmetric Gabor filter has the form:

$$
\begin{aligned}
G(x, y ; f, \theta) & =\exp \left\{-\frac{1}{2}\left[\frac{x^{\prime 2}}{\sigma_{x^{\prime}}^{2^{\prime}}}+\frac{y^{\prime 2}}{\sigma_{y^{\prime}}^{2^{\prime}}}\right]\right\} \cos 2 \pi f x^{\prime} \\
x^{\prime} & =x \sin \theta+y \cos \theta \\
y^{\prime} & =x \cos \theta-y \sin \theta
\end{aligned}
$$

where $f$ is the frequency of the sinusoidal plane wave along the direction $\theta$ from the $x$-axis, and $\sigma_{x}^{\prime}$ and $\sigma_{y}^{\prime}$ are the space constants of the Gaussian envelope along $x_{0}$ and $y_{0}$ axes, respectively. 
- A matrix of values $V(k, \theta)$ is obtained by computing the Absolute Average Distance (AAD) on each sector of the filtered images $T_{k \theta}$.

$$
V(i, \theta)=\frac{1}{n_{k}}\left(\sum_{1}^{n_{j}}\left|T_{k \theta}(x, y)-P_{k \theta}\right|\right),
$$

where $n_{k}$ is the number of pixels in $S_{k}$ and $P_{k \theta}$ is the mean of pixel values $T_{k \theta}(x, y)$ in the sector $S_{k}$.

For each minutia, the obtained feature vector is composed by $N_{F}=N_{S} \times N_{F}$ values. Considering that the template Fingercode is not rotational-invariant, we computed the feature matrix $F_{A}$ by according the $x_{0}$ and $y_{0}$ axes to the value of median ridge orientation $\beta$.

The matrix $H_{A}$ is obtained by computing a set of Histogram of Oriented Gradients (HOG) features in a fixed size area $\left(N_{p} \times N_{p}\right.$ pixels) around each minutia point $i$. The computation of the HOG features is performed by applying the method described in [19]. HOG features are gradient features designed for general object recognition. This method can be divided in the sequent steps.

- The gradient module image $G_{M}(x, y)$ and the gradient phase image $G_{P}(x, y)$ of the image $I$ are computed.

- The images $G_{M}(x, y)$ and $G_{P}(x, y)$ are divided into $c_{w} \times$ $c_{h}$ cells.

- At each cell, the orientation $\tilde{G}_{p}(x, y)$ is quantized into $c_{b}$ orientation bins, weighted by its magnitude $G_{M}(x, y)$.

- The histogram with the $c_{b}$ orientations is computed for each cell.

For each minutia $i, c_{w} \times c_{h} \times c_{b}$ features are obtained.

\section{Creation of the classification systems}

In order to compare the effectiveness of the extracted feature set, we tested various families of classification systems, in particular the Linear Bayes Normal Classifier (LDC), the family of the k-Nearest Neighbor classifier with odd values of the parameter $\mathrm{k}(1,3,5)$, the Linear classifier by KL expansion of common covariance matrix (KLLDC), the Linear classifier by PCA expansion on the joint data (PCA-LDC), Feed Forward neural networks with different number of neurons $\mathrm{Nr}$ in the hidden layer (FF-Nr). In this work, we considered neural networks with a two-layered topology where the hidden nodes are log-sigmoidal and the output node is linear. The classical backpropagation algorithm had been used as the training method. All tested classifiers had been validated with the $\mathrm{N}$-fold cross validation technique with $N=10$ [20].

\section{EXPERIMENTAL RESUlts}

\section{A. Experimental Settings}

The presented method had been written in Matlab language (Version 7.6) exploiting the available toolboxes on a Intel Centrino 2.0Ghz working with Windows XP Professional. The parameters used for the computation of the features related to the template Fingercode are: $N_{R}=2 ; N_{A}=4$; and $N_{F}=4$. The parameters used to the HOG features are: $c_{w}=3 ; c_{h}=3$; $c_{b}=9$.

(a)

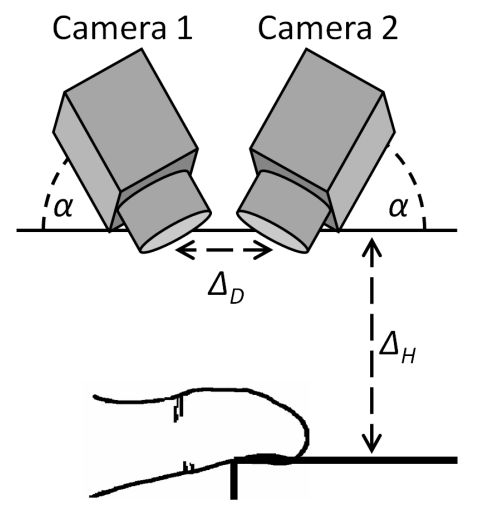

(c)

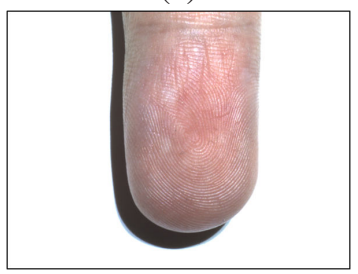

Fig. 4. Image acquisition: (a) schema of the acquisition setup; (b) example of image captured from Camera 2; (c) example of image captured from Camera 1.

\section{B. Datasets and Final Results}

The proposed method had been tested on images captured in our laboratory since, at best of our knowledge, there are not available any public datasets collecting two-view touchless fingerprint images. The image dataset is composed by 120 color images related to two different fingers, captured by two Sony SX90CR CCD cameras with different angles and distances. The size of each image is $1280 \times 960$ pixels, the illumination is controlled by four white LEDs and the used focal is $25 \mathrm{~mm}$. We used three different acquisition setup characterized by different angles between the cameras and the reference plane $(\alpha)$, distances between the centers of the optics $\left(\Delta_{D}\right)$, and distances from the finger to the optics $\left(\Delta_{H}\right)$.

- Setup 05: $\alpha=5^{\circ}, \Delta_{D}=45 \mathrm{~mm}$, and $\Delta_{H}=230 \mathrm{~mm}$;

- Setup 10: $\alpha=10^{\circ}, \Delta_{D}=75 \mathrm{~mm}$, and $\Delta_{H}=230 \mathrm{~mm}$;

- Setup 15: $\alpha=15^{\circ}, \Delta_{D}=125 \mathrm{~mm}$, and $\Delta_{H}=230 \mathrm{~mm}$.

For each setup, we collected 10 pairs of images related to each finger.

Fig. 4 shows the image acquisition schema and an example of captured fingerprint images.

We also collected a set of calibration images for each acquisition setup due to obtain a three-dimensional reconstruction of the minutiae points. We captured 15 different pairs of chessboard images for each setup. The used calibration chessboard is composed by $12 \times 9$ squares of $2,8 \times 2.8 \mathrm{~mm}$. Considering these images, we estimated a reconstruction error of the chessboards in the three-dimensional space equal to $0.03 \mathrm{~mm}$. This error is computed by considering the Euclidean distance of the corner points of each image to equidistant points belonging to interpolated planes. 
As a reference, we manually matched the minutiae present in a circle area with radius equal to 120 pixels around to the the core point of the fingerprint images. We considered a total of 24258 classified minutiae pairs, which are classified as positive (real pairs) and negative (false pairs).

For each image acquisition setup, we considered six different feature sets, obtaining 24 different datasets. For each minutiae pair $(i, j)$ of two fingerprint images $A$ and $B$, these datasets contain the following values.

- Feature set A: the Euclidean distance between $F_{A}(i)$ and $F_{B}(j)$;

- Feature set B: the Euclidean distance between $H_{A}(i)$ and $H_{B}(j)$;

- Feature set C: $M_{A}(i)-M_{B}(j)$, and the Euclidean distance between $F_{A}(i)$ and $F_{B}(j)$;

- Feature set D: $M_{A}(i)-M_{B}(j)$, and the Euclidean distance between $H_{A}(i)$ and $H_{B}(j)$;

- Feature set E: $M_{A}(i)-M_{B}(j)$, and $F_{A}(i)-F_{B}(j)$;

- Feature set F: $M_{A}(i)-M_{B}(j)$, and $H_{A}(i)-H_{B}(j)$.

The proposed method showed a remarkable accuracy, especially when the neural networks are adopted as the final classifiers of the system. Experiments showed the possibility to suitable train the neural networks in order to produce a correctly behavior with different setup configurations. Remarkably, the neural networks achieved a similar (or better) accuracy of the best traditional inductive method among the set we considered in our tests, and, most of the time, with a minor computational complexity.

In particular, Table I shows the results of different compositions of the presented features for the Setup 05. The proposed method achieves a good accuracy especially using features related to the euclidean distance between the features processed by Gabor filters (Dataset 05-C) with a classification mean error equal to $0.9 \%$. A similar situation is present in the experiments related to the Setup 10 and Setup 15. In particular, Table II reports that the neural networks obtained the best accuracy with a classification error of $1.5 \%$ in the Setup 15 by using the Dataset 15-A and Dataset 05-C. The only classification family showing a similar accuracy (on the considered datasets) are the kNN classifiers, but the neural network approach offers a relevant gain in the computational complexity. Within the presented experiments, the minimum gain factor found is more than 100 .

Experiments showed that the proposed sets of feature are capable to correctly classify the minutiae pairs with an interesting accuracy in the case of multiple images of the same finger and that the neural-based quality classification system is probably the most suitable model for real-time applications.

A quality analysis of the obtained three-dimensional minutiae sets for each image shows the effectiveness of the proposed reconstruction. The calibration procedure of the system, obtained by $2.8 \times 2.8 \mathrm{~mm}$ chessboards, showed a remarkable three-dimensional reconstruction accuracy with localization errors of few less then one tenth of millimeters. Fig. 5 plots an example of the reconstructed three-dimensional minutiae above the corresponding left input image. Vertical

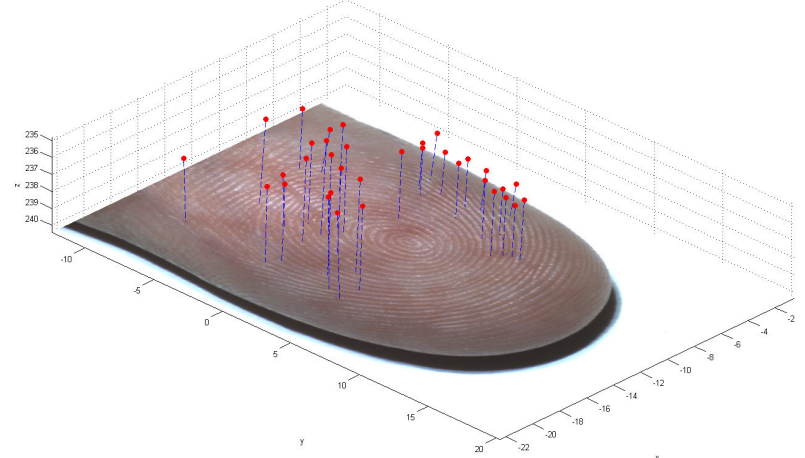

Fig. 5. Example of the reconstructed three-dimensional minutiae with the proposed system. Vertical segments show the correspondence between the identified three-dimensional points and the relative position of the minutiae on the left image. The distances are related to the reference view point and are expressed in $\mathrm{mm}$.

segments show the correspondences between the identified three-dimensional points and the relative position of the minutiae on the left image.

Classification errors produce mismatches in the minutiae pairs, hence, as a consequence, the resulting three-dimensional points tend to be quite far from the finger surface. This kind of points can be further post-processed and deleted from the minutiae list by using a simple three-dimensional spike filter.

Preliminary results on the use of three-dimensional minutiae captured from stereoscopic contact-less images shows that the final accuracy can be improved with respect to the use of single contact-less images, but larger datasets and further studies are needed to confirm this observation.

\section{CONCLUSIONS}

The paper proposed a new method to identify pairs of minutiae between two views of the same finger. This task is an important step in the 3D reconstruction of the fingerprint minutiae and in multiple views systems designed for contactless fingerprint images. The method is as follows. After an enhancement step of the input image, the candidate minutiae are selected in two fingerprint images by a classical method, and a list of candidate pairs is created. The proposed system builds a set of local features centered around the selected minutiae and then a classifier based on a trained neural network estimates if each candidate pair is valid or not. The output of the system is the list of the minutiae pairs present in the input images. In this context, the generalization capability of the neural network permits to efficiently and effectively learn the complex relationship needed to classify if a pair of minutiae extracted from two different images belongs to the same real minutiae point of the finger. Experiments showed that the method is feasible and accurate also in different light conditions and hardware configurations.

\section{ACKNOWLEDGMENT}

The work described in this paper has been partially supported by the EU within the 7FP project "PrimeLife" under grant agreement 216483 . 
TABLE I

RESULTS OBTAINED WITH THE $05^{\circ}$ DASETS

\begin{tabular}{r|cc|cc|cc|cc|cc|cc} 
& \multicolumn{2}{|c|}{ Dataset 05-A } & \multicolumn{2}{|c|}{ Dataset 05-B } & \multicolumn{2}{|c|}{ Dataset 05-C } & \multicolumn{2}{c|}{ Dataset 05-D } & \multicolumn{2}{c}{ Dataset 05-E } & \multicolumn{2}{c}{ Dataset 05-F } \\
Method & Mean & Std & Mean & Std & Mean & Std & Mean & Std & Mean & Std & Mean & Std \\
\hline linear & 0.0100 & 0.0000 & 0.0170 & 0.0000 & 0.0090 & 0.0010 & 0.0150 & 0.0000 & 0.0370 & 0.0000 & 0.0380 & 0.0000 \\
quadratic & 0.0100 & 0.0000 & 0.0180 & 0.0000 & 0.0080 & 0.0000 & 0.0080 & 0.0000 & 0.0120 & 0.0010 & 0.0360 & 0.0010 \\
pcldc & 0.0100 & 0.0000 & 0.0170 & 0.0000 & 0.0090 & 0.0000 & 0.0150 & 0.0010 & 0.0370 & 0.0000 & 0.0380 & 0.0010 \\
klldc & 0.0100 & 0.0000 & 0.0170 & 0.0000 & 0.0090 & 0.0000 & 0.0150 & 0.0000 & 0.0370 & 0.0000 & 0.0380 & 0.0000 \\
kNN-1 & 0.0180 & 0.0010 & 0.0310 & 0.0010 & 0.0110 & 0.0010 & 0.0160 & 0.0010 & 0.0130 & 0.0010 & 0.0160 & 0.0010 \\
kNN-3 & 0.0120 & 0.0000 & 0.0220 & 0.0010 & 0.0100 & 0.0010 & 0.0140 & 0.0010 & 0.0140 & 0.0010 & 0.0150 & 0.0010 \\
kNN-5 & 0.0110 & 0.0010 & 0.0210 & 0.0010 & 0.0100 & 0.0000 & 0.0130 & 0.0010 & 0.0150 & 0.0000 & 0.0140 & 0.0000 \\
kNN-10 & 0.0110 & 0.0010 & 0.0200 & 0.0010 & 0.0090 & 0.0010 & 0.0150 & 0.0000 & 0.0200 & 0.0000 & 0.0150 & 0.0000 \\
FNN-1 & 0.0150 & 0.0160 & 0.0260 & 0.0150 & 0.0080 & 0.0040 & 0.0140 & 0.0060 & 0.0400 & 0.0160 & 0.0650 & 0.0200 \\
FNN-3 & 0.0100 & 0.0060 & 0.0180 & 0.0100 & 0.0070 & 0.0030 & 0.0150 & 0.0100 & 0.0490 & 0.0160 & 0.0630 & 0.0140 \\
FNN-5 & 0.0100 & 0.0050 & 0.0190 & 0.0070 & 0.0100 & 0.0030 & 0.0130 & 0.0080 & 0.0420 & 0.0250 & 0.0560 & 0.0260 \\
FNN-10 & 0.0100 & 0.0060 & 0.0200 & 0.0090 & 0.0140 & 0.0120 & 0.0080 & 0.0060 & 0.0300 & 0.0180 & 0.0490 & 0.0200
\end{tabular}

Notes. Classification methods: Linear Classifier (linear); Quadratic Classifier (quadratic); Linear Classifier using PC expansion (pcldc); Linear Classifier using KL expansion (klldc); kNN with k=1 (kNN-1); kNN with k=3 (kNN-3); kNN with k=5 (kNN-5); kNN with k=10 (kNN-10); Feed-Foreword Neural Network with one hidden layer composed by 1 nodes (NN-1); Feed-Foreword Neural Network with one hidden layer composed by 3 nodes (NN-3); Feed-Foreword Neural Network with one hidden layer composed by 5 nodes (NN-5); Feed-Foreword Neural Network with one hidden layer composed by 10 nodes (NN-10).

TABLE II

RESULTS OBTAINED WITH THE $15^{\circ}$ DASETS

\begin{tabular}{r|cc|cc|cc|cc|cc|cc} 
& \multicolumn{2}{|c|}{ Dataset 15-A } & \multicolumn{2}{|c|}{ Dataset 15-B } & \multicolumn{2}{|c|}{ Dataset 10-C } & \multicolumn{2}{c|}{ Dataset 15-D } & \multicolumn{2}{c}{ Dataset 15-E } & \multicolumn{2}{c}{ Dataset 15-F } \\
Method & Mean & Std & Mean & Std & Mean & Std & Mean & Std & Mean & Std & Mean & Std \\
\hline linear & 0.0160 & 0.0010 & 0.0180 & 0.0000 & 0.0200 & 0.0000 & 0.0180 & 0.0010 & 0.0440 & 0.0010 & 0.0520 & 0.0020 \\
quadratic & 0.0200 & 0.0000 & 0.0170 & 0.0000 & 0.0180 & 0.0000 & 0.0150 & 0.0010 & 0.0400 & 0.0010 & 0.1460 & 0.0070 \\
pcldc & 0.0160 & 0.0010 & 0.0180 & 0.0010 & 0.0200 & 0.0000 & 0.0170 & 0.0010 & 0.0430 & 0.0000 & 0.0520 & 0.0020 \\
klldc & 0.0160 & 0.0010 & 0.0170 & 0.0000 & 0.0200 & 0.0000 & 0.0180 & 0.0010 & 0.0440 & 0.0000 & 0.0520 & 0.0020 \\
kNN-1 & 0.0230 & 0.0010 & 0.0310 & 0.0020 & 0.0270 & 0.0020 & 0.0200 & 0.0020 & 0.0170 & 0.0000 & 0.0190 & 0.0010 \\
kNN-3 & 0.0210 & 0.0020 & 0.0200 & 0.0020 & 0.0210 & 0.0010 & 0.0170 & 0.0010 & 0.0220 & 0.0020 & 0.0200 & 0.0020 \\
kNN-5 & 0.0180 & 0.0020 & 0.0170 & 0.0010 & 0.0150 & 0.0010 & 0.0200 & 0.0010 & 0.0270 & 0.0030 & 0.0190 & 0.0020 \\
kNN-10 & 0.0140 & 0.0000 & 0.0150 & 0.0010 & 0.0150 & 0.0010 & 0.0190 & 0.0000 & 0.0300 & 0.0020 & 0.0180 & 0.0010 \\
FNN-1 & 0.0240 & 0.0200 & 0.0240 & 0.0220 & 0.0200 & 0.0150 & 0.0200 & 0.0170 & 0.0590 & 0.0240 & 0.0650 & 0.0150 \\
FNN-3 & 0.0160 & 0.0130 & 0.0160 & 0.0090 & 0.0190 & 0.0090 & 0.0220 & 0.0180 & 0.0670 & 0.0220 & 0.0690 & 0.0290 \\
FNN-5 & 0.0150 & 0.0120 & 0.0170 & 0.0090 & 0.0150 & 0.0120 & 0.0180 & 0.0110 & 0.0780 & 0.0370 & 0.0500 & 0.0170 \\
FNN-10 & 0.0150 & 0.0140 & 0.0190 & 0.0170 & 0.0260 & 0.0150 & 0.0230 & 0.0230 & 0.0440 & 0.0340 & 0.0960 & 0.1260
\end{tabular}

Notes. Classification methods: Linear Classifier (linear); Quadratic Classifier (quadratic); Linear Classifier using PC expansion (pcldc); Linear Classifier using KL expansion (klldc); kNN with k=1 (kNN-1); kNN with k=3 (kNN-3); kNN with k=5 (kNN-5); kNN with k=10 (kNN-10); Feed-Foreword Neural Network with one hidden layer composed by 1 nodes (NN-1); Feed-Foreword Neural Network with one hidden layer composed by 3 nodes (NN-3); Feed-Foreword Neural Network with one hidden layer composed by 5 nodes (NN-5); Feed-Foreword Neural Network with one hidden layer composed by 10 nodes (NN-10).

\section{REFERENCES}

[1] G. Parziale, E. Diaz-Santana, and R. Hauke, "The surround imager: A multi-camera touchless device to acquire $3 \mathrm{~d}$ rolled-equivalent fingerprints," in Advances in Biometrics, ser. Lecture Notes in Computer Science, D. Zhang and A. Jain, Eds. Springer Berlin / Heidelberg, 2005, vol. 3832, pp. 244-250.

[2] Y. Wang, L. Hassebrook, and D. Lau, "Noncontact, depth-detailed 3d fingerprinting," vol. Newsroom, 2009.

[3] V. Piuri and F. Scotti, "Fingerprint biometrics via low-cost sensors and webcams," in 2nd IEEE International Conference on Biometrics: Theory, Applications and Systems, BTAS 2008., October 2008, pp. 1-6.

[4] B. Hiew, A. Teoh, and Y. Pang, "Touch-less fingerprint recognition system," in IEEE Workshop on Automatic Identification Advanced Technologies, June 2007, pp. 24-29.

[5] C. I. Watson, M. D. Garris, E. Tabassi, C. L. Wilson, R. M. McCabe, S. Janet, and K. Ko, "User's guide to nist biometric image software (NBIS)," National Institute of Standards and Technology, January 2007, http://fingerprint.nist.gov/NFIS.

[6] N. Ratha, K. Karu, S. Chen, and A. Jain, "A real-time matching system for large fingerprint databases," IEEE Transactions on Pattern Analysis and Machine Intelligence, August 1996.

[7] G. Bebis, T. Deaconu, and M. Georgiopoulos, "Fingerprint identification using delaunay triangulation," in International Conference on Information Intelligence and Systems, 1999, pp. 452-459.

[8] H. Khazaei and A. Mohades, "Fingerprint matching algorithm based on voronoi diagram," International Conference on Computational Science and its Applications, pp. 433-440, 2008.
[9] R. Cappelli, M. Ferrara, and D. Maltoni, "Minutia cylinder-code: A new representation and matching technique for fingerprint recognition," IEEE Transactions on Pattern Analysis and Machine Intelligence, vol. 32, pp. 2128-2141, 2010

[10] F. Benhammadi, M. N. Amirouche, H. Hentous, K. Bey Beghdad, and M. Aissani, "Fingerprint matching from minutiae texture maps," Pattern Recognition, vol. 40, no. 1, pp. 189-197, 2007.

[11] A. Ceguerra and I. Koprinska, "Automatic fingerprint verification using neural networks," in Proceedings of the International Conference on Artificial Neural Networks, ser. ICANN '02. Springer-Verlag, 2002, pp. 1281-1286.

[12] A. Arrieta, G. Estrada, L. Romero, and n. Lancho, "Neural networks applied to fingerprint recognition," in Distributed Computing, Artificial Intelligence, Bioinformatics, Soft Computing, and Ambient Assisted Living, ser. Lecture Notes in Computer Science, S. Omatu, M. Rocha, J. Bravo, F. Fernández, E. Corchado, A. Bustillo, and J. Corchado, Eds. Springer Berlin / Heidelberg, 2009, vol. 5518, pp. 621-625.

[13] C.-T. Hsieh and C.-S. Hu, "An application of fuzzy logic and neural network to fingerprint recognition," in Proceedings of the 4th WSEAS International Conference on Telecommunications and Informatics. Stevens Point, Wisconsin, USA: World Scientific and Engineering Academy and Society (WSEAS), 2005, pp. 1-6.

[14] K. N. Mutter, Z. M. Jafri, and A. A. Aziz, "Automatic fingerprint identification using gray hopfield neural network improved by run-length encoding," International Conference on Computer Graphics, Imaging and Visualization, pp. 205-210, 2008.

[15] C. Yu, Z. Jian, Y. Bo, and C. Deyun, "A novel principal component analysis neural network algorithm for fingerprint recognition in online examination system," Asia-Pacific Conference on Information Process- 
ing, vol. 1, pp. 182-186, 2009.

[16] R. Donida Labati, A. Genovese, V. Piuri, and F. Scotti, "Measurement of the principal singular point in contact and contactless fingerprint images by using computational intelligence techniques," in IEEE International Conference on Computational Intelligence for Measurement Systems and Applications (CIMSA), September 2010, pp. 18-23.

[17] L. Hong, Y. Wan, and A. Jain, "Fingerprint image enhancement: Algorithm and performance evaluation," IEEE Transactions on Pattern Analysis and Machine Intelligence, vol. 20, pp. 777-789, 1998.

[18] A. Jain, S. Prabhakar, L. Hong, and S. Pankanti, "Filterbank-based fingerprint matching," Image Processing, IEEE Transactions on, vol. 9, no. 5, pp. 846-859, May 2000.

[19] D. G. Lowe, "Distinctive image features from scale-invariant keypoints," International Joint Conference on Computer Vision, vol. 60, pp. 91-110, November 2004

[20] R. O. Duda, P. E. Hart, and D. G. Stork, Pattern Classification (2nd Edition), 2nd ed. Wiley-Interscience, November 2000. 